Check for updates

Cite this: Analyst, 2019, 144, 1147

\title{
Rapid and simple detection of ascorbic acid and alkaline phosphatase via controlled generation of silver nanoparticles and selective recognition $\uparrow$
}

\author{
Piaopiao Chen, ${ }^{a}$ Shixin Yan, ${ }^{a}$ Erica Sawyer, ${ }^{a}$ Binwu Ying, ${ }^{a}$ Xiawei Wei, ${ }^{a}$ \\ Zhengzhi Wu*b,c,d and Jia Geng (D)*a
}

\begin{abstract}
Ascorbic acid (AA) and alkaline phosphatase (ALP) serve as an important coenzyme and enzyme in multiple biological metabolism reactions, respectively, and abnormal levels of these substrates have been associated with several diseases. Herein, a new and simple fluorescence strategy has been developed for AA and ALP sensing by exploiting CdTe quantum dots (QDs) as an effective signal indicator. This method is mainly based on the selective fluorescence-quenching reaction between $\mathrm{Ag}^{+}$and $\mathrm{CdTe}$ QDs, as opposed to silver nanoparticles (Ag NPs); $\mathrm{Ag}^{+}$can be reduced to Ag NPs by AA. Furthermore, by taking advantage of AA as a mediator, this strategy is further exploited for ALP assay given that ALP can cause the hydrolysis of L-ascorbic acid-2-phosphate (AAP), which yields AA. Under optimal conditions, controlled generation of Ag NPs and the selective recognition-based sensing system exhibit high sensitivity toward $\mathrm{AA}$ and ALP with limits of detection (LODs) of $3 \mu \mathrm{M}$ and $0.25 \mathrm{UL}^{-1}$ and linear ranges of detection from 0 to $800 \mu \mathrm{M}$ and 1 to $1000 \mathrm{U} \mathrm{L}^{-1}$, respectively. Moreover, the sensor was successfully used for assaying AA in fruit juice and ALP in human serum. The results demonstrate that the proposed fluorescence strategy has significant advantages, such as its simplicity, cost-effectiveness, and rapid runtime, and the operational convenience of this label-free method further demonstrates its potential for constructing effective sensors with biochemical and clinical applications.
\end{abstract}

Received 8th October 2018, Accepted 17th November 2018

DOI: 10.1039/c8an01925h

rsc.li/analyst colorimetric, ${ }^{6}$ electrochemical, ${ }^{7}$ magnetic relaxation switching (MRS), ${ }^{8}$ surface-enhanced Raman scattering (SERS), ${ }^{9}$ and so on. Among these methods, the fluorescence strategy has significant advantages due to its ease of use, high sensitivity, rapid analysis, and good reproducibility. Consequently, it has received extensive attention. To date, many fluorescence methods were developed for AA and ALP detection using materials, such as carbon dots (CDs) ${ }^{10}$ cerium metal-organic frameworks (MOFs), ${ }^{11}$ gold nanoclusters (Au NCs),${ }^{12}$ quantum dots (QDs), ${ }^{13}$ DNA-templated silver nanoclusters (Ag NCs), ${ }^{14}$ poly(thymine)-templated copper nanoclusters ( $\mathrm{Cu} \mathrm{NCs}),{ }^{15}$ $\mathrm{MnO}_{2}$ nanosheets, ${ }^{16}$ etc.

Semiconductor QDs exhibit unique properties, e.g., width, symmetry, size-tunable emission spectra, broad absorption spectra, long photoluminescence lifetimes, and significant photo-stability, which allow for fascinating applications in sensing, bioimaging, etc. ${ }^{17,18}$ Additionally, cation exchange reactions have led to the development of a series of research methods for quantitative assays, ${ }^{19}$ which can be used to analyze the characteristics of nucleic acids ${ }^{20}$ proteins ${ }^{21}$ single-nucleotide polymorphisms (SNPs), ${ }^{22}$ and other substrates. These reaction conditions are mild, and strong acids and complex steps were not involved. ${ }^{23,24}$ Subsequently, researchers discovered that CdTe QDs can selectively dis-

\footnotetext{
${ }^{a}$ Department of Laboratory Medicine, State Key Laboratory of Biotherapy, West China Hospital, Sichuan University and Collaborative Innovation Center for Biotherapy, Chengdu, Sichuan, 610041, China. E-mail: geng.jia@scu.edu.cn

${ }^{b}$ The First Affiliated Hospital of Shenzhen University, Shenzhen, 518035, China ${ }^{c}$ The Eighth Affiliated Hospital of Sun Yat-sen University, Shenzhen, 518033, China ${ }^{d}$ Shenzhen Institute of Geriatrics, Shenzhen, 518020, China.

E-mail: szwzz001@email.szu.edu.cn

$\dagger$ Electronic supplementary information (ESI) available. See DOI: 10.1039/ c8an01925h
} 


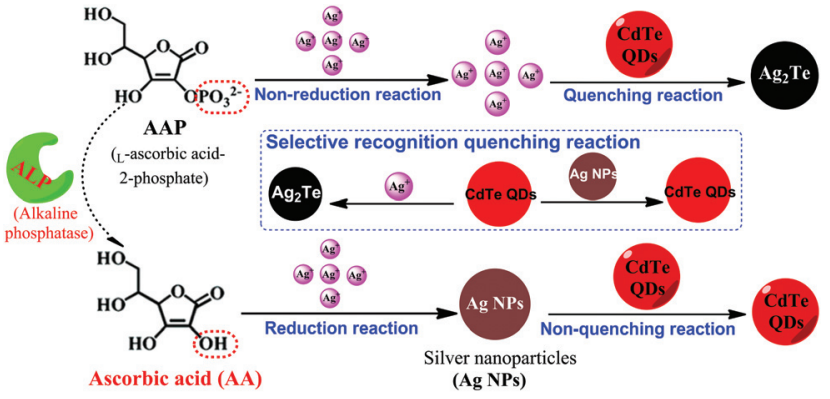

Scheme 1 Schematic illustration of the fluorescence AA and ALP assay based on the controlled generation of silver nanoparticles and selective quenching reaction.

tinguish $\mathrm{Ag}^{+}$from $\mathrm{Ag}$ NPs. Various detection methods have been developed based on this phenomenon. ${ }^{25}$ However, the selective recognition reaction of CdTe QDs has not been used for assaying AA and ALP.

In this study, by combining the reduction reactionmediated formation of Ag NPs and the selective recognition reaction between $\mathrm{Ag}^{+}$and CdTe QDs, a novel and simple sensor was designed for the highly sensitive assay of AA and ALP. AA can reduce $\mathrm{Ag}^{+}$to form Ag NPs, inhibiting the occurrence of a fluorescence quenching reaction, leading to the high fluorescence intensity of CdTe QDs. Therefore, the detection of AA was achieved using a fluorescence turn-on mode strategy. Furthermore, ALP enables the removal of $\mathrm{PO}_{4}{ }^{3-}$ from L-ascorbic acid-2-phosphate, which does not act as a reducing agent, and yields AA, which can then reduce $\mathrm{Ag}^{+}$to $\mathrm{Ag}$ NPs. Therefore, with AAP as a bridge, assay of ALP was successfully realized (as shown in Scheme 1). Subsequently, this sensing strategy was used for the analysis of AA (fruit juice) and ALP (human serum) in real samples, and satisfactory results were achieved. The proposed strategy of AA and ALP activity possesses advantages such as its ease of use, cost-effectiveness, convenience, and sensitivity.

\section{Experimental}

\subsection{Materials and instruments}

Silver nitrate $\left(\mathrm{AgNO}_{3}\right)$, glycine (Gly), glucose, ascorbic acid (AA), L-ascorbic acid 2-phosphate trisodium salt (AAP), alkaline phosphatase (ALP), human serum albumin (HSA), transferrin, trypsin, pepsin, papain, and thrombin were purchased from Sigma-Aldrich (St Louis, MO, USA). High purity $\mathrm{NaNO}_{3}, \mathrm{Mg}$ $\left(\mathrm{NO}_{3}\right)_{2}, \mathrm{HCl}, \mathrm{NaOH}, \mathrm{CdCl}_{2}, \mathrm{KBH}_{4}$, and trisodium citrate were obtained from Kelong Reagent Factory (Chengdu, China). 3-[NMorpholino] propanesulfonic acid (MOPS) was ordered from Solarbio Technology Co., Ltd (Beijing, China). $\mathrm{Na}_{2} \mathrm{TeO}_{3}$ and 3-mercaptopropionic acid (MPA) were obtained from Aladdin Reagent Co., Ltd (Shanghai, China). A MOPS buffer solution (10 mM, pH 7.0, $100 \mathrm{mM} \mathrm{NaNO}, 2.5 \mathrm{mM} \mathrm{Mg}\left(\mathrm{NO}_{3}\right)_{2}$ ) was used in this work. High purity deionized water $(18.2 \mathrm{M} \Omega \mathrm{cm}$,
Chengdu Pure Technology Co., Chengdu, China) was used in this work. The commercial ALP kit was obtained from Roche Pharmaceuticals (Germany). Human serum samples were provided by the Department of Laboratory Medicine, West China Hospital of Sichuan University (Chengdu, China).

The F-7100 spectrometer (Hitachi, Japan) was used to record the emission spectra and the signal of CdTe QDs at a $365 \mathrm{~nm}$ excitation wavelength. Absorption spectra of CdTe QDs were recorded on a Hitachi U-1750 UV-vis spectrophotometer (Shimadzu, Kyoto, Japan). High-resolution transmission electron microscopy (HR-TEM) measurements of CdTe QDs were carried out using a Tecnai G2F20 STWIN TEM at an accelerating voltage of $200 \mathrm{kV}$ (FEI Co., USA).

\subsection{Synthesis of CdTe QDs}

A one-pot synthetic method was adopted to obtain CdTe QDs with slight modifications. ${ }^{26}$ Firstly, a $50 \mathrm{~mL}$ solution containing $\mathrm{CdCl}_{2}(0.5 \mathrm{mmol})$ and trisodium citrate $(0.2 \mathrm{~g})$ was prepared. Then, MPA $(52 \mu \mathrm{L})$ was instantly added into the above solution, and the solution $\mathrm{pH}$ was adjusted to 10.5 using $\mathrm{NaOH}$. Later, $\mathrm{Na}_{2} \mathrm{TeO}_{3}(0.1 \mathrm{mmol})$ and $\mathrm{KBH}_{4}(50 \mathrm{mg})$ were added into the above solution, and then this solution mixture was refluxed for $1 \mathrm{~h}$ to obtain the CdTe QDs solution. Subsequently, high purity CdTe QDs were obtained via precipitation with $n$-propanol and centrifugation (11000 rpm). The purified CdTe QDs were redispersed in high purity water before use. ${ }^{27}$ The fluorescence and UV-vis absorption spectra were recorded (Fig. 1C). The MPA-CdTe QDs were kept at $4^{\circ} \mathrm{C}$.

\subsection{AA assay}

As per the sensing assay of AA shown in Scheme 1, different concentrations of AA $(50 \mu \mathrm{L})$ were mixed with $\mathrm{Ag}^{+}(50 \mu \mathrm{L}, 5 \mu \mathrm{M})$ in $100 \mu \mathrm{L}$ MOPS buffer in the dark at room temperature (RT)

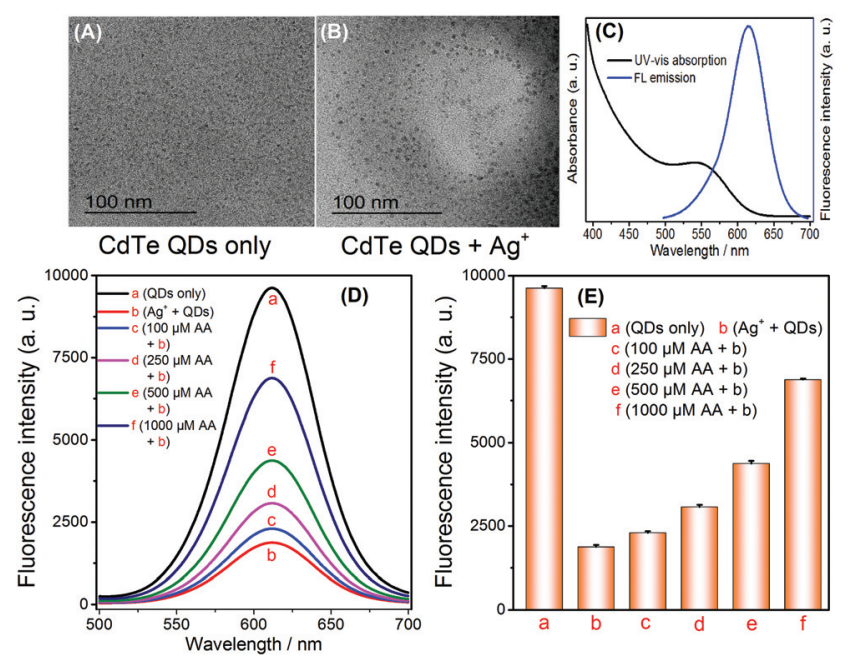

Fig. 1 TEM images of CdTe QDs before (A) and after (B) the addition of $\mathrm{Ag}^{+}$in the quenching reaction. (C) The spectra of the UV-vis absorption (black curve) and the fluorescence emission ( $F L$, blue curve) of the CdTe QDs. (D) and (E) Both show the feasibility of the AA assay based on the controlled generation of Ag NPs and selective quenching reaction. 
for $20 \mathrm{~min}$ to form silver nanoparticles (Ag NPs). The same reaction mixtures without the target $\mathrm{AA}$ were used as negative controls. Then, $10 \mu \mathrm{L}$ CdTe QDs (a 10-fold dilution of the original CdTe QDs solution) was mixed with the above solution and further incubated in the dark for $10 \mathrm{~min}$ at RT. Finally, the resulting solution was diluted to $1 \mathrm{~mL}$ and fluorescence measurements were performed immediately. All of the measurements were performed at least three times.

\subsection{ALP assay}

The sensitive fluorescence detection of ALP started with the addition of $50 \mu \mathrm{L}$ AAP $(1 \mathrm{mM})$ and $50 \mu \mathrm{L}$ ALP (various concentrations), which were added into MOPS buffer $(100 \mu \mathrm{L})$. Later, the above solution mixtures were incubated for $30 \mathrm{~min}$ at $37{ }^{\circ} \mathrm{C}$. Then, $\mathrm{Ag}^{+}$solution $(5 \mu \mathrm{M}, 50 \mu \mathrm{L})$ was added into the mixtures, which were further incubated in the dark at RT for $20 \mathrm{~min}$ to form Ag NPs. Then, $10 \mu \mathrm{L}$ CdTe QDs (a 10-fold dilution of the original CdTe QDs solution) was added into the above solution and further maintained in the dark for $10 \mathrm{~min}$ at RT. Finally, the resulting solution was diluted to $1 \mathrm{~mL}$ and the fluorescence measurements (with excitation at $365 \mathrm{~nm}$ ) were performed immediately. All of the measurements were performed at least three times.

\subsection{Real sample preparation}

The fruit juice sample was diluted 100-fold with high purity water before analysis.

Healthy human serums were prepared with a 50-fold dilution with high purity water, and then filtered using a $30 \mathrm{kDa}$ ultrafiltration membrane to remove the interfering macromolecules and small molecules. The ultrafiltrates were spiked with $\operatorname{ALP}\left(1,10,100\right.$, and $1000 \mathrm{U} \mathrm{L} \mathrm{L}^{-1}$, final concentrations). For the ALP assay, the spiked diluted serum samples served as the standard solutions as described in the ALP assay procedure.

\section{Results and discussion}

\subsection{Sensing principle}

The principle of our proposed selective fluorescence quenching reaction-based strategy for AA and ALP sensing is illustrated in Scheme 1. Its mechanism is mainly based on $\mathrm{Ag}^{+}$ being reduced by AA to generate $\mathrm{Ag}$ NPs, and the ability of CdTe QDs to selectively differentiate $\mathrm{Ag}^{+}$and $\mathrm{Ag}$ NPs. In the absence of the corresponding targets, $\mathrm{Ag}^{+}$can effectively quench the fluorescence of CdTe QDs by a rapid cation exchange reaction and an obvious reduction in the fluorescence signal can be recorded. However, in the presence of the target AA, Ag NPs can be formed from $\mathrm{Ag}^{+}$via $\mathrm{AA}$ reduction. The CdTe QDs stay intact and have a strong fluorescence signal. Because the increasing degree of the fluorescence signal is proportional to the concentration of $\mathrm{AA}$, target AA can be successfully detected through the fluorescence change of the CdTe QDs. Furthermore, by taking advantage of $\mathrm{AA}$ as a mediator, this sensing platform was extended to ALP detection due to the ability of ALP to catalyze the hydrolysis of AAP to yield AA. Therefore, the AA and ALP assay used a turn-on mode strategy, and the concentration of CdTe QDs was proportional to the amount of AA and ALP; this enabled the conversion of the target reaction events into a detectable signal of the CdTe QDs via a fluorescence spectrometer.

\subsection{The feasibility of the AA assay}

In accordance with the principle shown in Scheme 1, the feasibility of AA sensing was investigated. The TEM images of the CdTe QDs and the products from the cation exchange reaction indicate that the spherical CdTe QDs (Fig. 1A) become $\mathrm{Ag}_{2} \mathrm{Te}$ aggregates (Fig. 1B), thus verifying the cation exchange reaction between CdTe QDs and $\mathrm{Ag}^{+}$. To further confirm the feasibility of this strategy, AA of different concentrations (100, 250, $500,1000 \mu \mathrm{M})$ were added into the $\mathrm{Ag}^{+}$solution. After the addition of CdTe QDs the selective quenching reaction was triggered, and the fluorescence signal changes were recorded. As shown in Fig. 1D and E, the fluorescence signal of CdTe QDs increased significantly when the concentration of AA increased (Fig. 1D-b vs. 1D-c, d, e, f). The above results indicate that AA can reduce $\mathrm{Ag}^{+}$and inhibit the quenching of CdTe QDs. Thus, the feasibility of this technique has been demonstrated for AA sensing while displaying its advantages, such as its simple operation and rapid response.

\subsection{Detection of AA}

To ensure the strategy presented in this work for sensitive detection of AA, the sensing conditions were first optimized. $\mathrm{An}^{+} \mathrm{Ag}^{+}$volume of $50 \mu \mathrm{L}(5 \mu \mathrm{M})$ (Fig. S1 $\left.\dagger\right)$, a 20 min duration of $\mathrm{Ag}$ NPs formation by AA reduction of $\mathrm{Ag}^{+}$(Fig. S2 $\dagger$ ), and a $10 \mathrm{~min}$ duration for the selective quenching reaction (Fig. S3†) were finally adopted for subsequent experiments. Under the optimized conditions, when the concentration of AA increased, the fluorescence intensity also increased (Fig. 2A). Fig. 2B describes the relationship between the signal enhancement and the concentrations of AA; the inset of Fig. 2B shows a good linearity $\left(R^{2}=0.992\right)$ from 0 to $800 \mu \mathrm{M}$ with a LOD of $3 \mu \mathrm{M}(3 \sigma, n=11)$. The comparison of AA detection with other methods is summarized (Table 1). Although our method does not have the highest sensitivity, it has the advantages of simplicity in both design and operation, as well as its low cost. In addition, a series of eleven repetitive measurements of $100 \mu \mathrm{M}$ AA were used for estimating the precision of this method, and the resulting RSD value was $2.9 \%$.

Selectivity was investigated by challenging this fluorescence strategy with potential interferents, including abundant cations $\left(\mathrm{K}^{+}, \mathrm{Na}^{+}, \mathrm{Ca}^{2+}, \mathrm{Mg}^{2+}, \mathrm{Ba}^{2+}, \mathrm{Zn}^{2+}, \mathrm{Ni}^{2+}, \mathrm{Al}^{3+}\right)$, glucose, and glycine (Gly). As shown in Fig. 2C and D, $100 \mu \mathrm{M}$ AA resulted in the obvious enhancement of fluorescence. In contrast, no obvious change in fluorescence was monitored by adding $100 \mu \mathrm{M}$ of the other analytes. Therefore, the high selectivity of the sensor could be attributed to the selective quenching reaction between $\mathrm{Ag}^{+} / \mathrm{Ag}$ NPs and CdTe QDs and the reduction ability of AA towards $\mathrm{Ag}^{+}$. 

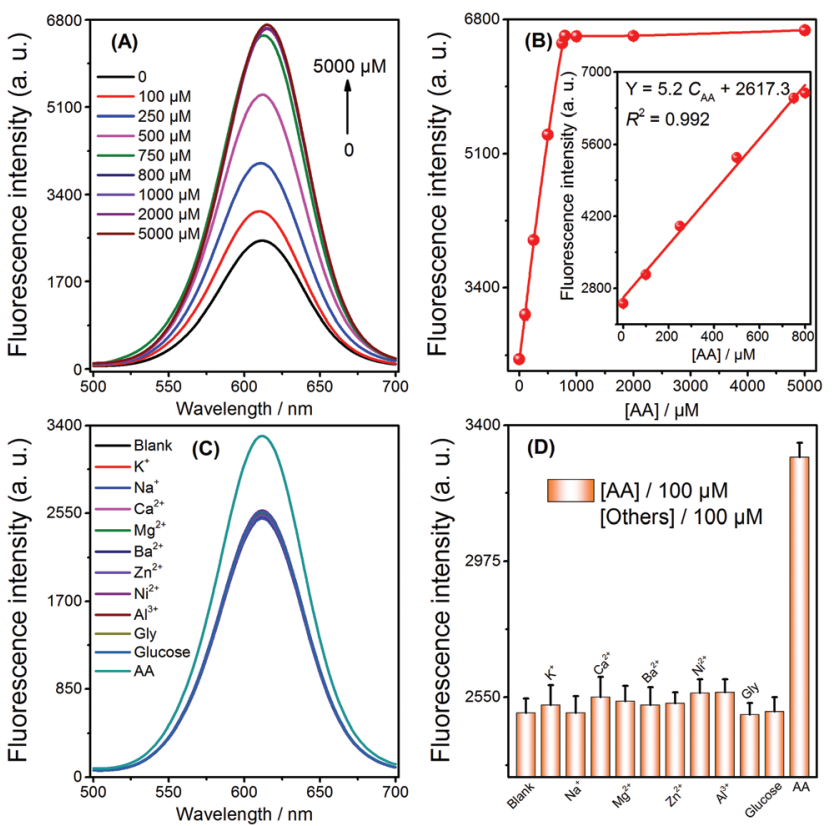

Fig. 2 The analytical performance and selectivity of this sensor for AA detection. (A) and (B) Fluorescence spectra and calibration curve for AA detection. Inset: A linear relationship of the fluorescence signal versus the concentration of $A A$ ranging from 0 to $800 \mu \mathrm{M}$. (C) and (D) Fluorescence spectra and fluorescence signal of different interferents. All concentrations were $100 \mu \mathrm{M}$. Error bars were estimated from at least three replicate measurements.

Table 1 Comparison of different fluorescence strategies for the detection of $A A$

\begin{tabular}{|c|c|c|c|}
\hline Method & Linear range & LOD & Ref. \\
\hline $\mathrm{Au} \mathrm{NCs}{ }^{a}$ & $5-100 \mu \mathrm{M}$ & $5 \mu \mathrm{M}$ & 28 \\
\hline $\mathrm{Au}$ NCs-PbS-QDs ${ }^{b}$ & $3-40 \mu \mathrm{M}$ & $1.5 \mu \mathrm{M}$ & 12 \\
\hline GQDs $^{c}$ & $1-30 \mu \mathrm{M}$ & $270 \mathrm{nM}$ & 29 \\
\hline $\mathrm{MOF}^{d}$ & $0-80 \mu \mathrm{M}$ & $7 \mathrm{nM}$ & 11 \\
\hline $\mathrm{CoOOH}$-modified TPNPs ${ }^{e}$ & $1-20 \mu \mathrm{M}$ & $170 \mathrm{nM}$ & 30 \\
\hline $\mathrm{UCNPs}^{f}$ & $0.1-25 \mu \mathrm{M}$ & $0.04 \mu \mathrm{M}$ & 31 \\
\hline $\mathrm{CD}-\mathrm{CoOOH}^{g}$ & $0.1-20 \mu \mathrm{M}$ & $50 \mathrm{nM}$ & 10 \\
\hline N-CNPs ${ }^{h}$ & $0.2-150 \mu \mathrm{M}$ & $50 \mathrm{nM}$ & 32 \\
\hline CdTe QDs & $0-800 \mu \mathrm{M}$ & $3 \mu \mathrm{M}$ & This work \\
\hline
\end{tabular}

${ }^{a}$ Gold nanoclusters. ${ }^{b}$ Gold nanoclusters and NIR PbS QDs. ${ }^{c}$ Graphene quantum dots. ${ }^{d}$ Cerium metal-organic framework (ZJU-136-Ce). ${ }^{e}$ Cobalt oxyhydroxide nanoflake two-photon nanoparticles. ${ }^{f}$ Upconversion nanoparticles. ${ }^{g}$ Carbon dot-modified hexagonal cobalt oxyhydroxide nanoflakes. ${ }^{h}$ Nitrogen-doped carbon nanoparticles.

The application of the fluorescent sensor for AA detection in $1 \%$ fruit juice was evaluated using the spiked method, and the resulting fluorescence spectra and signals were recorded (shown in Fig. 3). It is observed that the signal of the solution increased with the increasing concentration of AA from $0.5 \mu \mathrm{M}$ to $800 \mu \mathrm{M}$; a strong linear relationship was demonstrated in this range (Fig. 3B, inset). These results imply that the sensor is capable of being used to detect AA in real samples in a practical and efficient manner.
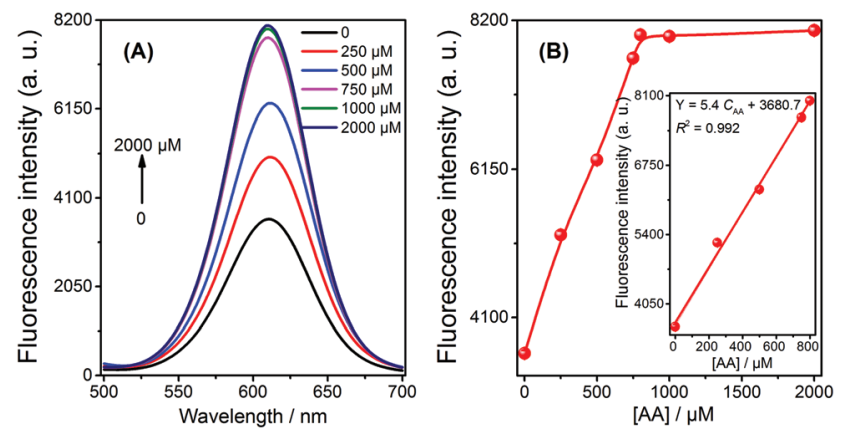

Fig. 3 The analytical performance of this ascorbic acid sensor in 1\% fruit juice solution. (A) Fluorescence emission spectra of the biosensor upon the addition of different concentrations of AA. (B) Calibration curve with the inset showing the linear relationship of the fluorescence signal versus the concentration of AA ranging from 0.5 to $800 \mu \mathrm{M}$. Error bars were estimated from three replicate measurements.

\subsection{Assay of ALP}

The results of the aforementioned experiments inspired the extension of applications for this method. Given that ALP can catalyze the hydrolysis of AAP to generate AA, this property enabled us to develop a novel strategy for assaying ALP via the selective quenching reaction. Before evaluating its analytical performance, the feasibility of the ALP fluorescence assay was verified. The fluorescence signal was obviously reduced after the addition of $\mathrm{Ag}^{+}$into the CdTe QDs solution, which indicated that the quenching reaction had been initiated (Fig. 4A-a vs. 4A-b). At the same time, no significant signal changes were observed when AAP was added to the above $\mathrm{Ag}^{+}+\mathrm{CdTe}$ QDs solution (Fig. 4A-b vs. 4A-c). Later, when different concentrations of ALP were added into the blank solutions, the fluorescence signals of the solutions gradually increased (Fig. 4A-d and A-e); this indicated that AA had been successfully generated and $\mathrm{Ag}^{+}$was reduced to $\mathrm{Ag}$ NPs. Overall, these results show that the strategy can be extended to the assay of ALP and the strategy outlined in Scheme 1 works well.

To test whether the strategy could be used for quantitative detection of target ALP, the fluorescence signals observed with various ALP concentrations were first measured to evaluate the

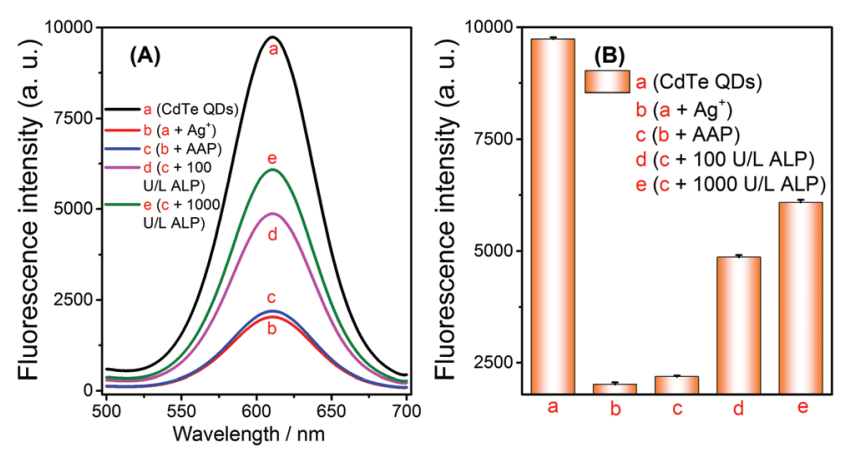

Fig. 4 Feasibility of the alkaline phosphatase fluorescence assay based on the controlled generation of silver nanoparticles and the selective quenching reaction. 
sensitivity of the sensor under the previously optimized conditions (shown in Fig. S4, see the ESI†े). The fluorescence signals of the biosensor increased with the increasing concentrations of ALP ranging from 1 to $1000 \mathrm{U} \mathrm{L}^{-1}$ (Fig. 5A). A calibration plot of ALP activity is shown in Fig. 5B (inset), and the correlation equation was $Y=1218 \log C_{\mathrm{ALP}}+3568$ ( $C$ is the concentration of ALP, $\left.R^{2}=0.993\right)$. The limit of detection can be reached at a low concentration of $0.25 \mathrm{U} \mathrm{L}^{-1}$, and the LOD of this ALP detection is comparable to that of other methods (Table 2). A series of eleven repetitive measurements of
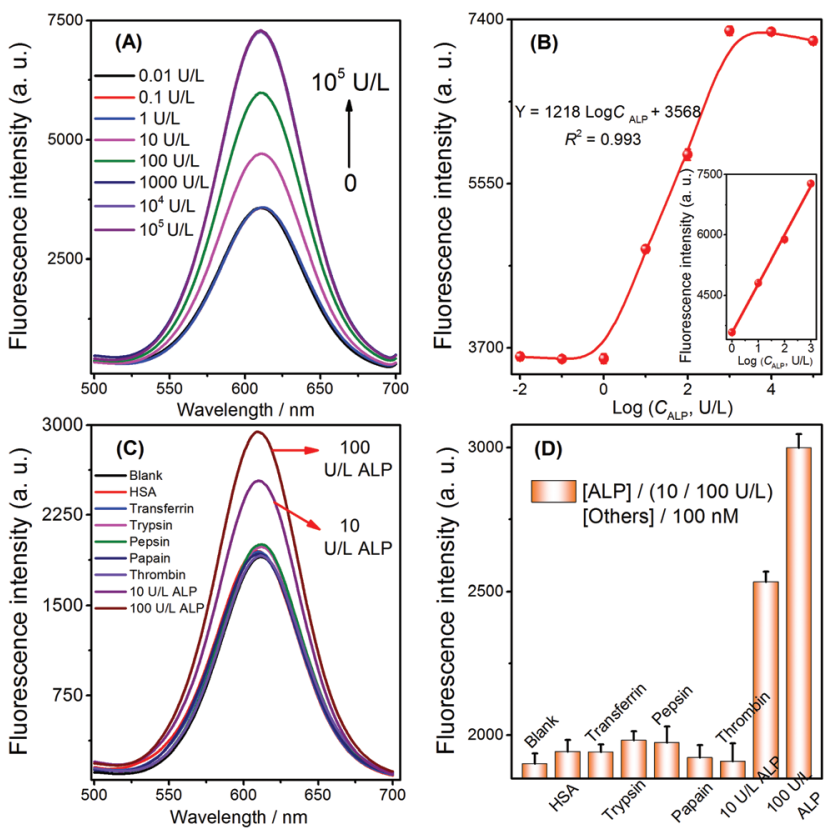

Fig. 5 The analytical performance and selectivity of this sensor for ALP detection. (A) Fluorescence spectra of the obtained CdTe QDs in the presence of ALP using different concentrations. (B) Calibration curve for ALP detection with the inset displaying the linearity of the fluorescence intensity with respect to logarithmic ALP concentrations. Fluorescence spectra (C) and the fluorescence signal (D) of the fluorescence assay of ALP $\left(10\right.$ and $\left.100 \mathrm{U} \mathrm{L}^{-1}\right)$ and interferents $(100 \mathrm{nM})$. Error bars were estimated from at least three replicate measurements.

Table 2 Comparison of different strategies for the detection of ALP

\begin{tabular}{lll}
\hline Method & Linear range; LOD & Ref. \\
\hline Fluorescence (PolyT-Cu NPs) & $0-70 \mathrm{U} \mathrm{L}^{-1} ; 1 \mathrm{U} \mathrm{L} \mathrm{L}^{-1}$ & 15 \\
Fluorescence (silver nanoclusters) & $30-240 \mathrm{U} \mathrm{L}^{-1} ; 5 \mathrm{U} \mathrm{L}^{-1}$ & 33 \\
Colorimetric (Au NPs) & $0-6.8 \mu \mathrm{M} ; \mathrm{NM}^{b}$ & 6 \\
Colorimetric (Au/Ag core/shell & $5-100 \mathrm{U} \mathrm{L}^{-1} ; 3.3 \mathrm{U} \mathrm{L}^{-1}$ & 34 \\
nanorods) & & \\
Electrochemical & $0.1-10 \mathrm{U} \mathrm{L}^{-1} ; 0.1 \mathrm{U} \mathrm{L}^{-1}$ & 35 \\
Photoelectrochemical & $0.5-40 \mathrm{U} \mathrm{L}^{-1} ; 0.33 \mathrm{U} \mathrm{L}^{-1}$ & 36 \\
Magnetic relaxation switching $_{\text {SERS }}$ & $29.3-3750 \mathrm{U} \mathrm{L}^{-1} ; 0.42 \mathrm{U} \mathrm{L}^{-1}$ & 8 \\
Fluorescence (CdTe QDs) & $0.72-3 \mathrm{U} \mathrm{L}^{-1} ; 0.01 \mathrm{U} \mathrm{L}^{-1}$ & 37 \\
& $1-1000 \mathrm{U} \mathrm{L}^{-1} ; 0.25 \mathrm{U} \mathrm{L}^{-1}$ & This \\
& & work
\end{tabular}

${ }^{a}$ Surface-enhanced Raman scattering. ${ }^{b}$ Not mentioned.
Table 3 Determination of ALP in human serum samples

\begin{tabular}{lcllll}
\hline Samples & $\begin{array}{l}\text { ALP kit } \\
\left(\mathrm{U} \mathrm{L}^{-1}\right)\end{array}$ & $\begin{array}{l}\text { This method } \\
\left(\mathrm{U} \mathrm{L}^{-1}\right)\end{array}$ & $\begin{array}{l}\text { Added, } \\
\mathrm{U} \mathrm{L}^{-1}\end{array}$ & $\begin{array}{l}\mathrm{Found}^{a}, \\
\mathrm{U} \mathrm{L}^{-1}\end{array}$ & $\begin{array}{l}\text { Recovery }^{b} \\
\%\end{array}$ \\
\hline 1 & 65 & 60 & 100 & $158 \pm 5$ & 98 \\
2 & 71 & 75 & 200 & $270 \pm 15$ & 97 \\
3 & 97 & 100 & 300 & $388 \pm 23$ & 96 \\
4 & 72 & 67 & 500 & $570 \pm 15$ & 100 \\
5 & 88 & 83 & 750 & $830 \pm 15$ & 99 \\
6 & 100 & 105 & 900 & $1000 \pm 23$ & 99
\end{tabular}

${ }^{a}$ Mean and standard deviation of results $(n=3) .{ }^{b}$ Recovery $(\%)=$ $\left(C_{\text {Found }} / C_{\text {Added }}\right) \times 100 \%$.

$100 \mathrm{U} \mathrm{L}^{-1}$ ALP were used for estimating the precision of this method, and the resulting RSD value was 3.6\%.

The selectivity of the selective quenching reaction-based strategy was investigated using pepsin, papain, thrombin, trypsin, transferrin, and human serum albumin as interfering substances. A significant increase in fluorescence was only observed with the target ALP (10 and $100 \mathrm{U} \mathrm{L}^{-1}$ ); in contrast, there was no increase observed when using the other compounds, which were employed at comparatively higher concentrations (100 nM, Fig. 5C and D). These experimental results indicated that the simple turn-on method possessed high specificity for the ALP assay, which was mainly due to the highly specific catalytic hydrolysis of AAP by ALP.

To evaluate the general applicability of the sensor to clinical samples, this sensor was used in a complex sample matrix of human serum. It is noteworthy that the interference from $\mathrm{Cu}^{2+}$ can be eliminated in real samples by using an ultrafiltration membrane in sample pretreatment. A similar signal curve was observed when the sensor was introduced to $2 \%$ serum in comparison with the curve of the buffer solution, which was observed under the same experimental conditions (Fig. S5†). Furthermore, the designed CdTe QDs-based method was used to detect the concentrations and recoveries of ALP by spiking 50 -fold dilutions of human serum samples with a series of known ALP concentrations. The experimental results of the two methods (our method and the commercial ALP kit colorimetric method) are comparable; moreover, satisfactory recoveries were obtained ( $96 \%$ to $100 \%$, Table 3$)$. Therefore, the above experimental results have demonstrated that this method has potential for practical sample analysis.

\section{Conclusions}

In summary, we have developed a simple and novel strategy for the detection of AA and ALP by exploiting CdTe QDs as an effective signal indicator. In the assay, the reduction reactionmediated formation of $\mathrm{Ag}$ NPs and the selective quenching reaction between $\mathrm{Ag}^{+} / \mathrm{Ag}$ NPs and CdTe QDs were utilized. This method has the following advantages: (1) mild reaction conditions without any rigorous operation; (2) rapid response (completes in a few minutes); and (3) good analytical performance, and it has been successfully applied to real samples. Given these advantages, 
this simple, new strategy may hold great potential for further applications in biomedical research and disease diagnosis.

\section{Conflicts of interest}

There are no conflicts to declare.

\section{Acknowledgements}

This work was supported by the National Key Research and Development Program of China (2016YFA0201400 and 2016YFC1200300) and the National Natural Science Foundation of China (81574038).

\section{Notes and references}

1 Q. Chen, M. G. Espey, A. Y. Sun, C. Pooput, K. L. Kirk, M. C. Krishna, D. B. Khosh, J. Drisko and M. Levine, Proc. Natl. Acad. Sci. U. S. A., 2008, 105, 11105-11109.

2 R. Heller, F. Münscher-Paulig, R. Gräbner and U. Till, J. Biol. Chem., 1999, 274, 8254-8260.

3 J. R. Eastman and D. Bixler, Clin. Chem., 1977, 23, 17691770.

4 S. G. Julien, N. Dubé, S. Hardy and M. L. Tremblay, Nat. Rev. Canacer, 2011, 11, 35-49.

5 M. C. Rong, L. P. Lin, X. H. Song, Y. R. Wang, Y. X. Zhong, J. W. Yan, Y. F. Feng, X. Y. Zeng and X. Chen, Biosens. Bioelectron., 2015, 68, 210-217.

6 Y. Choi, N. H. Ho and C. H. Tung, Angew. Chem., Int. Ed., 2007, 119, 721-723.

7 H. Wang, G. Q. Pu, S. Devaramani, Y. F. Wang, Z. F. Yang, L. F. Li, X. F. Ma and X. Q. Lu, Anal. Chem., 2018, 90, 48714877.

8 M. L. Dong, W. S. Zheng, Y. P. Chen, B. Ran, Z. Y. Qian and X. Y. Jiang, Anal. Chem., 2018, 90, 2833-2838.

9 P. Muhammad, X. Y. Tu, J. Liu, Y. J. Wang and Z. Liu, ACS Appl. Mater. Interfaces, 2017, 9, 12082-12091.

10 L. B. Li, C. Wang, K. Y. Liu, Y. H. Wang, K. Liu and Y. Q. Lin, Anal. Chem., 2015, 87, 3404-3411.

11 D. Yue, D. Zhao, J. Zhang, L. Zhang, K. Jiang, X. Zhang, Y. J. Cui, Y. Yang, B. L. Chen and G. D. Qian, Chem. Commun., 2017, 53, 11221-11224.

12 P. Zhao, K. Y. He, Y. T. Han, Z. Zhang, M. Z. Yu, H. H. Wang, Y. Huang, Z. Nie and S. Z. Yao, Anal. Chem., 2015, 87, 9998-10005.

13 L. Jia, J. P. Xu, D. Li, S. P. Pang, Y. Fang, Z. G. Song and J. Ji, Chem. Commun., 2010, 46, 7166-7168.

14 X. Q. Liu, F. A. Wang, A. Niazov-Elkan, W. W. Guo and I. Willner, Nano Lett., 2012, 13, 309-314.
15 J. Sun, T. Hu, X. L. Xu, L. Wang and X. R. Yang, Nanoscale, 2016, 8, 16846-16850.

16 T. Xiao, J. Sun, J. H. Zhao, S. Wang, G. Y. Liu and X. R. Yang, ACS Appl. Mater. Interfaces, 2018, 10, 6560-6569.

17 Y. F. Kong, J. Chen, H. W. Fang, G. Heath, Y. Wo, W. L. Wang, Y. X. Li, Y. Guo, S. D. Evans and S. Y. Chen, Chem. Mater., 2016, 28, 3041-3050.

18 J. Kim, M. J. Biondi, J. J. Feld and W. C. Chan, ACS Nano, 2016, 10, 4742-4753.

19 D. H. Son, S. M. Hughes, Y. D. Yin and A. P. Alivisatos, Science, 2004, 306, 1009-1012.

20 X. R. Zhang, H. X. Liu, R. J. Li, N. B. Zhang, Y. Xiong and S. Y. Niu, Chem. Commun., 2015, 51, 6952-6955.

21 Z. Z. Lin, S. Z. Lv, K. Y. Zhang and D. P. Tang, J. Mater. Chem. B, 2017, 5, 826-833.

22 X. R. Zhang, Y. Zhang, F. T. Chen, Y. Li and S. S. Zhang, Chem. Commun., 2016, 52, 13261-13264.

23 J. S. Li, T. R. Zhang, J. P. Ge, Y. D. Yin and W. W. Zhong, Angew. Chem., Int. Ed., 2009, 121, 1616-1619.

24 J. J. Yao, X. G. Han, S. Zeng and W. W. Zhong, Anal. Chem., 2012, 84, 1645-1652.

25 K. Huang, K. L. Xu, J. Tang, L. Yang, J. R. Zhou, X. D. Hou and C. B. Zheng, Anal. Chem., 2015, 87, 6584-6591.

26 Z. H. Sheng, H. Y. Han, X. F. Hu and C. Chi, Dalton Trans., 2010, 39, 7017-7020.

27 W. W. Yu, L. H. Qu, W. Z. Guo and X. G. Peng, Chem. Mater., 2003, 15, 2854-2860.

28 L. Z. Hu, L. Deng, S. Alsaiari, D. Y. Zhang and N. M. Khashab, Anal. Chem., 2014, 86, 4989-4994.

29 L. L. Feng, Y. X. Wu, D. L. Zhang, X. X. Hu, J. Zhang, P. Wang, Z. L. Song, X. B. Zhang and W. H. Tan, Anal. Chem., 2017, 89, 4077-4084.

30 H. M. Meng, X. B. Zhang, C. Yang, H. L. Kuai, G. J. Mao, L. Gong, W. H. Zhang, S. L. Feng and J. B. Chang, Anal. Chem., 2016, 88, 6057-6063.

31 Q. Q. Wu, H. Y. Chen, A. J. Fang, X. Y. Wu, M. L. Liu, H. T. Li, Y. Y. Zhang and S. Z. Yao, ACS Sens., 2017, 2, 18051813.

32 X. H. Zhu, T. B. Zhao, Z. Nie, Y. Liu and S. Z. Yao, Anal. Chem., 2015, 87, 8524-8530.

33 J. L. Ma, B. C. Yin, X. Wu and B. C. Ye, Anal. Chem., 2016, 88, 9219-9225.

34 Z. Q. Gao, K. C. Deng, X. D. Wang, M. Miró and D. P. Tang, ACS Appl. Mater. Interfaces, 2014, 6, 18243-18250.

35 L. F. Zhang, T. Hou, H. Y. Li and F. Li, Analyst, 2015, 140, 4030-4036.

36 N. Zhang, Y. F. Ruan, L. B. Zhang, W. W. Zhao, J. J. Xu and H. Y. Chen, Anal. Chem., 2018, 90, 2341-2347.

37 Y. Zeng, J. Q. Ren, S. K. Wang, J. M. Mai, B. Qu, Y. Zhang, A. G. Shen and J. M. Hu, ACS Appl. Mater. Interfaces, 2017, 9, 29547-29553. 